\title{
Evolutionary conservation and modulation of a juvenile growth-regulating genetic program
}

\author{
Angela Delaney ${ }^{1}$, Vasantha Padmanabhan ${ }^{3}$, Geoffrey Rezvani ${ }^{1}$, Weiping Chen ${ }^{2}$, \\ Patricia Forcinito', Crystal S F Cheung', Jeffrey Baron ${ }^{1,{ }^{*}}$ and Julian C K Lui ${ }^{1,{ }^{*}}$ \\ ${ }^{1}$ Program on Developmental Endocrinology and Genetics, Eunice Kennedy Shriver National Institute of \\ Child Health and Human Development ${ }^{2}$ Microarray Core Facility, National Institute of Diabetes and Digestive \\ and Kidney Diseases, National Institutes of Health, 10 Center Drive, MSC-1103; Building 10, Room 1-3330, Bethesda, \\ Maryland 20892, USA \\ ${ }^{3}$ Department of Pediatrics and the Reproductive Sciences Program, The University of Michigan, Ann Arbor, \\ Michigan, USA \\ *( J Baron and J C K Lui contributed equally to this work)
}

Correspondence should be addressed to J Baron

Email

jeffrey.baron@nih.gov

\begin{abstract}
Body size varies enormously among mammalian species. In small mammals, body growth is typically suppressed rapidly, within weeks, whereas in large mammals, growth is suppressed slowly, over years, allowing for a greater adult size. We recently reported evidence that body growth suppression in rodents is caused in part by a juvenile genetic program that occurs in multiple tissues simultaneously and involves the downregulation of a large set of growthpromoting genes. We hypothesized that this genetic program is conserved in large mammals but that its time course is evolutionarily modulated such that it plays out more slowly, allowing for more prolonged growth. Consistent with this hypothesis, using expression microarray analysis, we identified a set of genes that are downregulated with age in both juvenile sheep kidney and lung. This overlapping gene set was enriched for genes involved in cell proliferation and growth and showed striking similarity to a set of genes downregulated with age in multiple organs of the juvenile mouse and rat, indicating that the multiorgan juvenile genetic program previously described in rodents has been conserved in the 80 million years since sheep and rodents diverged in evolution. Using microarray and real-time $P C R$, we found that the pace of this program was most rapid in mice, more gradual in rats, and most gradual in sheep. These findings support the hypothesis that a growth-regulating genetic program is conserved among mammalian species but that its pace is modulated to allow more prolonged growth and therefore greater adult body size in larger mammals.
\end{abstract}
Key Words
- gene expression
- phylogeny
- growth limitation
- evolutionary conservation

Journal of Molecular

Endocrinology

(2014) 52, 269-277

\section{Introduction}

In the mammalian fetus, there is rapid proliferation in many tissues, driving rapid organ growth and consequently rapid growth in overall body size. However postnatally, this rapid growth slows progressively, causing body size eventually to plateau in adulthood. For example, in the human fetus, in mid-gestation, body length increases at the extraordinary rate of $100 \mathrm{~cm} /$ year, but then the linear growth rate declines to $\sim 50 \mathrm{~cm} /$ year by the time of birth, and subsequently to $5 \mathrm{~cm} /$ year by midchildhood (Tanner \& Davies 1985). In the human, but not 
in most other mammals, this pattern of deceleration is transiently interrupted by the pubertal growth spurt, when the linear growth rate increases temporarily to $\sim 10 \mathrm{~cm} /$ year, but then the overall decline resumes, until linear growth ceases by mid to late adolescence. This decline in linear growth primarily reflects the decreasing proliferation of growth plate chondrocytes, but similar declines in proliferation also occur in many other tissues, causing growth of other organs also to conclude by young adulthood (Winick \& Noble 1965, Chang et al. 2008).

This progressive deceleration in body growth appears to be caused primarily by local mechanisms, intrinsic to the growing tissues, rather than systemic mechanisms. Although hormones, such as growth hormone, insulinlike growth factor 1 (IGF1), and thyroid hormone, are potent regulators of body growth, their levels do not change with age in a pattern that could explain the declining growth rate (Kwan \& Hartman 2007). Furthermore, when juvenile organs are transplanted into adult recipients, their growth is maintained at juvenile rates, suggesting that this growth deceleration is dependent on factors intrinsic to the organ itself (Stevens et al. 1999, Pape et al. 2006, Schäfer et al. 2006).

We have recently shown evidence that, in the mouse and rat, growth deceleration is driven by a growthregulating genetic program, which involves the coordinated downregulation with age of many growth-promoting genes (Lui et al. 2008, 2010a, Finkielstain et al. 2009). This program occurs simultaneously in multiple organs, including the kidney, lung, liver, and heart, providing an explanation for the fact that growth deceleration occurs simultaneously in multiple organs throughout the body.

The mechanisms responsible for growth deceleration have been primarily studied in rodents; far less is known about growth regulation in large mammals. Like rodents, large mammals show rapid growth in early life followed by growth deceleration, but this decline occurs more gradually, allowing for more prolonged growth and therefore greater adult body size. In mice and rats, most body growth is complete by 2 months of age (Hughes \& Tanner 1970), whereas cows grow for $\sim 3$ years (Kratochvilova et al. 2002), and humans (Tanner \& Davies 1985) and elephants (Shrader et al. 2006) more than 10 years. From an evolutionary standpoint, the wide variation in the pace of growth deceleration presumably serves to achieve enormously divergent adult body sizes in different mammalian species. However, the mechanisms underlying this phylogenetic variation in growth patterns are unknown. In particular, it is unknown whether the multiorgan juvenile genetic program, which contributes to growth deceleration in the rodent, also serves to limit growth in large mammals.

We hypothesized that the growth-regulating genetic program identified in mice and rats has been conserved in larger mammals, but that, in larger mammals, it has been evolutionarily modulated to proceed more gradually, resulting in more prolonged growth and greater adult body size. To test this hypothesis, we compared gene expression patterns in the mouse, rat, and sheep.

\section{Materials and methods}

\section{Animal procedures}

C57BL/6 mice (Finkielstain et al. 2009, Lui et al. 2010a,b), Sprague-Dawley rats (Lui et al. 2010a), and Suffolk sheep (Manikkam et al. 2004) were maintained as previously described. Sheep (female) were killed with a barbiturate overdose (Fatal Plus, Vortech Pharmaceuticals, Dearborn, MI, USA), and tissues were obtained at fetal day 65 (FD65), FD90, 3.5 weeks postnatal, 10 weeks postnatal (after weaning at 8 weeks), 5 months (prepubertal, lung unavailable), and 21 months (young adult). Kidney, lung, liver, and heart were dissected ( $n=5$ animals per time point) and stored at $-80^{\circ} \mathrm{C}$. Mice were weighed before death and were killed by carbon dioxide inhalation at 1, 4, and 8 weeks and at 3, 9, and 15 months of age. Rats were killed by carbon dioxide inhalation at 1 and 5 weeks of age. Additional weight data for C57BL/ 6 mice (age 4-16 weeks, $n=100 /$ group) were obtained from Jackson Laboratory (Bar Harbor, ME, USA) and for Sprague-Dawley rats (age 3-12 weeks, $n=94$ /group) from Harlan Laboratories (Indianapolis, IN, USA). All animal procedures were approved by the National Institute of Child Health and Human Development Animal Care and Use Committee (mice and rats) and the University of Michigan Committee for the Use and Care of Animals (sheep).

\section{RNA extraction and purification}

Total RNA was extracted using TRIzol (Invitrogen) followed by RNeasy Mini Kit purification (Qiagen). For RNA extracted from lung, additional $\mathrm{LiCl}$ precipitation was carried out (Heinrichs et al. 1994). RNA integrity was confirmed using an Agilent 2100 Bioanalyzer (Agilent Technologies, Santa Clara, CA, USA).

\section{Expression microarray}

No ovine-specific microarray was available commercially. However, sheep and cows differ at $<3 \%$ of protein-coding

Published by Bioscientifica Ltd. 
nucleotides, and bovine microarrays have been used to study expression in the sheep (Diez-Tascon et al. 2005, Chen et al. 2007, MacKinnon et al. 2009). We therefore used GeneChip Bovine Genome Arrays (23,000 transcripts, Affymetrix, Santa Clara, CA, USA) to analyse the expression in kidney and lung obtained at FD90, 10 weeks, and 21 months of age ( $n=5$ per time point). Each microarrayanalysed RNA sample was isolated from a single animal. Microarray data have been deposited in the NCBI's Gene Expression Omnibus (GEO Series accession number: GSE48916). Microarray signals were analysed using the Affymetrix RMA algorithm. ANOVA and false discovery rate (FDR) analyses were carried out for probe sets using Partek Pro software (Partek, St Charles, MO, USA). Affymetrix ortholog tables were used to align microarray probes among species. For those genes represented by more than one probe set, we chose a single probe set for the analysis. Because some probe sets may show poor specificity for the gene of interest or poor sensitivity, either of which can obscure changes in expression, we included in the analysis those probe sets that showed the greatest temporal changes. Interspecies analyses involving sheep were limited to the 6825 genes present on all three ortholog tables for mouse, rat, and cow. Biological functions enriched in the commonly up- or downregulated gene sets in both organs in the sheep were assessed by Ingenuity Pathway Analysis (IPA) software 9.0 (Ingenuity Systems, Redwood City, CA, USA), using the 6825 genes as the reference/background gene set. Sheep results were compared with the 316 genes commonly regulated with age in multiple organs identified by microarray in mouse and rat (Finkielstain et al. 2009, Lui et al. 2010a,b). Heat maps were generated using JMP 8 software (SAS Institute, Cary, NC, USA). We repeated analyses after excluding genes that are known to be cell cycle related, based on GeneCards (www.genecards.org), the NCBI Gene database, and PubMed.

\section{Quantitative real-time RT-PCR}

Total RNA (1-2 $\mu \mathrm{g}, n=5$ samples per time point) was reverse transcribed using SuperScript III Reverse Transcriptase (Invitrogen). Quantitative real-time PCR was carried out in triplicate using the ABI prism 7900 Sequence Detection System (Applied Biosystems) and a VIC-labeled Taqman Assay (18S; catalog number 4319413E, Applied Biosystems) or custom primers (Ezh2, Gpc3, Igf2, Mest, Peg3, Plagl1, Smo; Invitrogen) and Power SYBR Green detection (Applied Biosystems). Primer design was based on Ovis aries sequence from NCBI Gene database (Igf2, Smo) or by sequencing cDNA (Ezh2, Gpc3, Mest, Peg3, Plagl1), and the primer sequences are as follows: Ezh2, forward primer (F): $5^{\prime}$-TGCTTCCTACATCGTAAGTGCA- ${ }^{\prime}$, reverse primer (R): 5'-TTGATGAAGATCCCCCAGC-3'; Gpc3， F: 5'-GCCAAGAACTACACCAATGCC-3', R: 5'-TCCCTTCTTCGGCTGGATAAG-3'; Igf2, F: 5'-TTCTTGCCTTCTTGGCCTTC-3', R: 5'-TTTATGCGGCTGGATGGTC-3'; Mest, F: 5'-ATCCCGAGTTTTTGGAGCTG-3', R: 5'-AGAAGGAGTTGATGAAGCCCA-3'; Peg3, F: 5'-ACCCGATTCAGGACAACATGG-3', R: 5'-AGGCGCTTCTCTTTGACCTTG-3'; Plagl1, F: 5'-CTGCCCCTCAAAACCGATACT-3', R: 5'-CCTCCCTTAGCCAGGTCAAAA-3'; Smo, F: 5'-TGAGCGGCATCTGTTTTGTG-3', R: 5'-GGTGGTTGCTCTTGATGGAGA-3' . Relative expression was calculated as: $2^{-\Delta C \mathrm{C}} \times 10^{6}$, where $C_{\mathrm{t}}=$ threshold cycle, $\Delta C_{\mathrm{t}}=\left(C_{\mathrm{t}}\right.$ of gene of interest $)-\left(C_{\mathrm{t}}\right.$ of $\left.18 \mathrm{~S} \mathrm{rRNA}\right)$. Results were compared with previously reported real-time RT-PCR data in mouse (Finkielstain et al. 2009, Lui et al. 2010a).

\section{Statistical analyses}

Data are mean \pm s.E.M. To determine whether genes up- or downregulated in one organ (or species) were similarly regulated in the second organ (or species), we used the $\chi^{2}$ test. For these tests, the expected number of genes commonly up- or downregulated in both organs (or both sets of species) was calculated based on the null hypothesis that the regulation in the two organs (or species) was independent and therefore that the frequency of genes upor downregulated in both was the product of the individual frequencies. In a second test to evaluate the similarities in age regulation between organs, genes were divided based on changes in expression in one sheep organ into agedownregulated, age-upregulated, and neither up- nor downregulated (significance regulation: FDR $<0.1$ ). The changes in gene expression in the second organ were then compared among the three groups. A similar analysis was carried out to compare changes in expression with age between the sheep and the multiorgan juvenile genetic program in the rodent. For this test, $P$ values were calculated by ANOVA on ranks using Dunn's method for multiple comparisons. For bioinformatic analyses, the probability that each biological function was due to chance was calculated using right-tailed Fisher's exact test, adjusted for multiple comparisons with the BenjaminiHochberg procedure. To compare the temporal changes in gene expression among species, relative expression was normalized to the young adult time point (mice, 8 weeks; sheep, 21 months). Age was expressed as weeks postfertilization to account for differences in gestation length (mouse, rat: 3 weeks; sheep: 21 weeks).

Published by Bioscientifica Ltd. 


\section{Results}

\section{Identification of genes that are regulated concordantly with age in both sheep lung and kidney}

We recently identified a multiorgan growth-regulating genetic program that occurs in mice and rats during juvenile growth deceleration (Finkielstain et al. 2009, Lui et al. 2010a). Here, we tested whether the program is also conserved in a large mammal, the sheep. Microarray analysis was used to compare gene expression between sheep at FD90, when body growth is rapid, 10 weeks postnatal, when growth is slowing, and 21 months, when body growth is complete, in kidney and lung. The analysis was limited to 6825 genes present on the ortholog tables for all three species studied. Of 1601 genes that were significantly downregulated with age in sheep kidney (criterion: $P<0.038$, ANOVA; FDR for probe sets $<0.1$ ), and 1787 genes that were downregulated in sheep lung $(P<0.044$, FDR $<0.1), 886$ genes were downregulated in both organs (Fig. 1A), an overlap far greater than expected

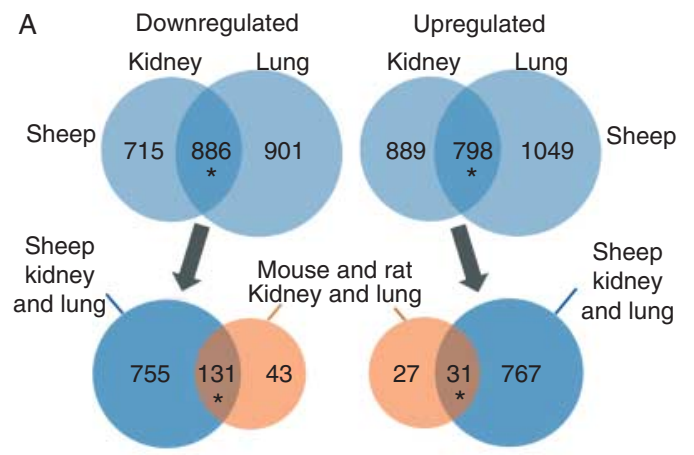

B

\begin{tabular}{|c|c|c|}
\hline \multicolumn{3}{|c|}{ Ingenuity pathway analysis } \\
\hline \multicolumn{3}{|c|}{ Commonly downregulated genes ( 886 genes) } \\
\hline Molecular function $(1)$ & $P$ value $^{(2)}$ & No. of genes ${ }^{(3)}$ \\
\hline DNA replication, recombinaion, and repair & $9.90 \times 10^{-12}$ & 183 \\
\hline Cell cycle & $4.98 \times 10^{-6}$ & 211 \\
\hline Cellular assembly and organization & $4.98 \times 10^{-6}$ & 183 \\
\hline Cell morphology & $5.99 \times 10^{-5}$ & 107 \\
\hline Cellular function and maintenance & $5.99 \times 10^{-5}$ & 155 \\
\hline Molecluar transport & $8.01 \times 10^{-5}$ & 34 \\
\hline RNA trafficking & $8.01 \times 10^{-5}$ & 18 \\
\hline Gene expression & $2.10 \times 10^{-4}$ & 211 \\
\hline Celluar compromise & $6.84 \times 10^{-4}$ & 36 \\
\hline Celluar development & $4.32 \times 10^{-3}$ & 268 \\
\hline Celluar growth and proliferation & $4.32 \times 10^{-3}$ & 327 \\
\hline \multicolumn{3}{|c|}{ Commonly upregulated genes (798 genes) } \\
\hline Molecular function & $P$ value & No. of genes \\
\hline Celluar function and maintenance & $2.34 \times 10^{-10}$ & 211 \\
\hline Celluar development & $2.56 \times 10^{-10}$ & 156 \\
\hline Celluar growth and proliferation & $2.56 \times 10^{-10}$ & 133 \\
\hline Cell-to-cell signaling and interaction & $5.17 \times 10^{-10}$ & 158 \\
\hline Protein synthesis & $1.15 \times 10^{-8}$ & 102 \\
\hline Cell death and survival & $1.65 \times 10^{-7}$ & 288 \\
\hline Cellular compromise & $1.65 \times 10^{-7}$ & 68 \\
\hline Molecular transport & $9.50 \times 10^{-7}$ & 204 \\
\hline Cell signaling & $7.87 \times 10^{-7}$ & 74 \\
\hline Vitamin and mineral metabolism & $7.87 \times 10^{-6}$ & 65 \\
\hline Cellular movement & $9.10 \times 10^{-6}$ & 163 \\
\hline Cell morphology & $1.01 \times 10^{-5}$ & 104 \\
\hline
\end{tabular}

C

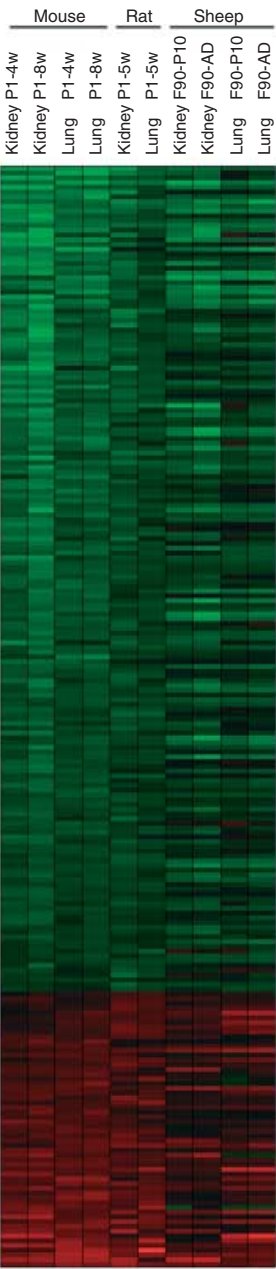

D
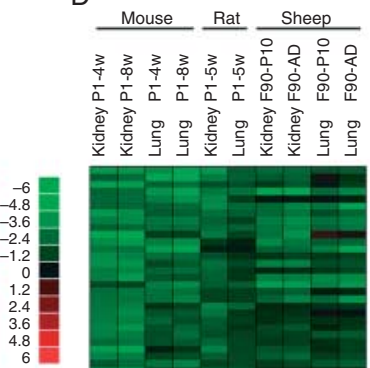

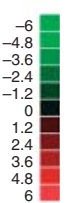

Figure 1

A multiorgan growth-regulating genetic program common to mice, rats, and sheep. (A) Venn diagrams showing the number of genes significantly downregulated and upregulated with age by microarray in sheep vs mouse and rat. Genes that showed significant age regulation (FDR $<0.1)$ in both kidney and lung in sheep were compared with the 174 genes downregulated or the 58 genes upregulated in all organs studied in mouse and rat. ${ }^{*} P<0.001$. (B) Gene ontology analysis of the genes that were age-regulated in both organs in sheep (FDR $<0.1)$. Downregulated (886) and upregulated (798) genes were analysed separately using Ingenuity Pathway Analysis. Only cellular and molecular functions with $P<0.005$

http://jme.endocrinology-journals.org DOI: 10.1530/JME-13-0263
(C) 2014 Society for Endocrinology Printed in Great Britain after Benjamini-Hochberg correction are listed. (C) Heat map based on microarray analysis of the 232 genes that were commonly down- or upregulated with age in the mouse (columns 1-4) and rat (columns 5-6) and present in the sheep microarray (columns 7-10). Each row corresponds to a gene, ranked by average fold change in mouse and rat. (D) Heat map similar to (C) but excluding 78 genes that have a known function specific to the cell cycle. Green, downregulation with age; red, upregulation. Scale values are $\log _{2}$ (fold difference). P, postnatal age (weeks); F, fetal age (days); $A D$, adult (21 months). 
by chance $\left(P<0.001, \chi^{2}\right.$ test $)$. Similarly, 1687 genes were upregulated in kidney $(P<0.038, \mathrm{FDR}<0.1), 1847$ genes were upregulated in lung $(P<0.044, \mathrm{FDR}<0.1)$, and 798 genes were upregulated in both organs with age (Fig. 1A), again a greater overlap than expected by chance $\left(P<0.001, \chi^{2}\right.$ test $)$. In addition, genes that were downregulated in sheep kidney also tended to be downregulated in sheep lung $(P<0.001$, Supplementary Fig. $1 \mathrm{~A}$, see section on supplementary data given at the end of this article), whereas genes that were upregulated in sheep kidney also tended to be upregulated in sheep lung $(P<0.001$, Supplementary Fig. 1A).

The biological functions of the genes that were commonly down- or upregulated ( $P$ values as above) in both organs were assessed by bioinformatic analysis (IPA 9.0). For both the down- and upregulated genes, this analysis identified an overrepresentation of genes involved in the cell cycle and in cellular growth and proliferation (Fig. 1B).

\section{The multiorgan juvenile genetic program previously identified in rodents is conserved in sheep}

We previously identified an extensive genetic program that involves the downregulation of many growth-promoting genes during juvenile life in the mouse and rat. This program occurs in multiple organs, including the kidney, lung, liver, and heart (Lui et al. 2008, 2010a, Finkielstain et al. 2009). To determine whether this multiorgan juvenile genetic program also occurs in sheep, we analysed the 174 genes that were significantly downregulated in all organs investigated in mouse and rat (Finkielstain et al. 2009, Lui et al. 2010a) and for which orthologs could be identified in all three species. Of these 174 genes, 131 genes (75\%) were also significantly downregulated with age $(\mathrm{FDR}<0.1)$ in both kidney and lung of the sheep (Fig. 1A and Supplementary Table 1 , see section on supplementary data given at the end of this article). This overlap was far greater than expected by chance $\left(P<0.001, \chi^{2}\right.$ test). Of the 58 genes that were significantly upregulated in all organs investigated in the mouse and rat, 31 genes (53\%) were significantly upregulated with age in both organs in the sheep, again more than expected by chance $(P<0.001)$. As a second analysis of evolutionary conservation, we constructed a heat map to visualize changes in expression of the 232 genes that were commonly down- or upregulated in all organs investigated in both the mouse and rat and that have identifiable orthologs in all three species. A striking similarity in the changes in gene expression with age was seen in the sheep (Fig. 1C). In a third analysis of evolutionary conservation, we found that downregulated genes in the multiorgan genetic program previously described in rodents tended to be downregulated in sheep lung and kidney $(P<0.001$ each, Supplementary Fig. 1B).

For subsequent analyses, we focused on those genes that were downregulated with age because prior bioinformatic and experimental functional studies in mice and rats indicated that the downregulated set and not the upregulated set was enriched for genes related to cell proliferation and growth (Lui et al. 2010a). Some of these age-downregulated genes might encode regulatory proteins that drive the decline in cell proliferation, and thus are of great interest, whereas other downregulated genes might play an obligatory role in the cell cycle machinery and thus their declining expression might represent less interesting markers of declining proliferation. We therefore repeated our analyses excluding genes with a known function specific to the cell cycle. The number of genes downregulated with age in all organs studied in all three species remained significantly greater than expected by chance $(P<0.001)$. A heat map of these non-cell-cycle-related genes revealed similar temporal expression patterns in sheep and rodents (Fig. 1D). Taken together, these findings suggest that the multiorgan growth-regulating genetic program identified in the mouse and rat is evolutionarily conserved in the sheep.

\section{The pace of the multiorgan juvenile genetic program differs among species of different body size}

Adult body size varies greatly among mammalian species. This evolutionary diversity is achieved in part by variation in the duration of embryonic and juvenile growth. In mice, body growth is mostly complete by 8 weeks after fertilization. In rats the decline occurs more slowly, such that growth is mostly complete by 11 weeks after fertilization, yielding an adult body mass $\sim 20$-fold greater than that of mice. In sheep, body growth slows even more gradually, with substantial growth persisting 11 months after fertilization, resulting in a body size 2000 -fold greater than that of mice (Fig. 2).

We hypothesized that the more gradual deceleration in juvenile body growth in larger mammals reflects a more gradual decline in the expression of growth-promoting genes in the juvenile multiorgan program. Consistent with this hypothesis, among genes downregulated in all organs studied in mouse and rat, the relative expression showed a greater fold decline from 1 to 4 weeks of age in the mouse, than from 1 to 5 weeks in the rat, despite the greater time interval for rats, in both kidney $(P<0.001)$

Published by Bioscientifica Ltd. 


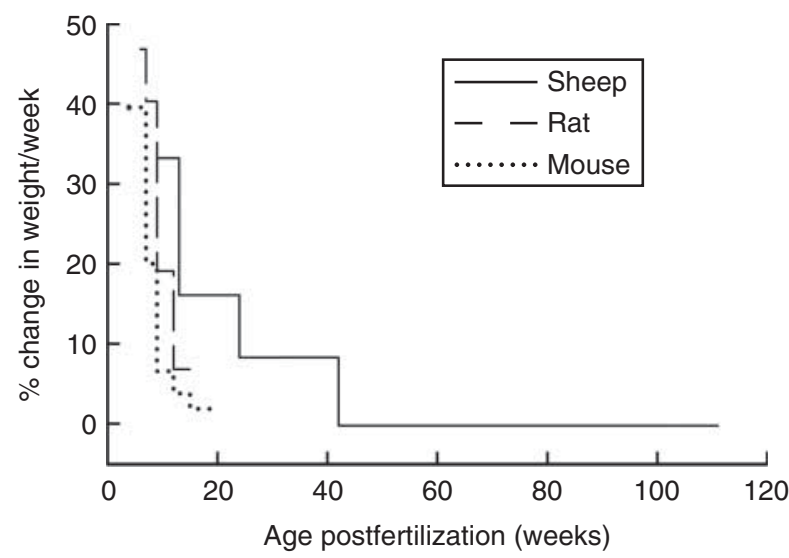

Figure 2

Rate of decline in growth velocity varies among mouse, rat, and sheep. Percent change in body weight per change in time was calculated for mouse (dotted line) based on weights at 4, 7, 9, 12, 15, and 19 weeks postfertilization; rat (dashed line) at $6,7,9,12$, and 15 weeks postfertilization; and sheep (solid line) at 9, 13, 24, 31, 42, and 111 weeks postfertilization. Age is expressed as weeks postfertilization to account for interspecies differences in gestational age.

and lung $(P<0.001)$ (Fig. 3A). For the 81 commonly upregulated genes no significant differences were observed between species (data not shown). In sheep, the decline in expression occurred even more slowly. Among genes downregulated in all organs studied in all three species, substantial declines persisted in sheep beyond 31 weeks postfertilization, whereas declines in the mouse were largely complete by 11 weeks postfertilization (Fig. 3B). To focus on genes that are more likely to physiologically govern proliferation rates, we repeated these analyses excluding genes with a functional role specific to the cell cycle. Sheep kidney showed a yet more gradual decline, whereas median expression did not decline in sheep lung (data not shown).

To characterize further the time course of downregulation in mammals of differing body size, we compared age-related changes in expression between mouse and sheep by real-time RT-PCR. We focused on genes that promote growth, as evidenced by the fact that targeted ablation diminishes body growth, and particularly on regulatory genes, such as genes encoding transcription factors or proteins involved in growth factor signaling. The mRNA expression of all seven genes studied (Ezh2, Gpc3, Igf2, Mest, Peg3, Plagl1, Smo) declined with age in kidney, lung, liver, and heart in both species (Fig. 4). In mouse, the declines were largely completed by 7 weeks postfertilization. In contrast, in sheep, expression continued to decrease until at least 31 weeks postfertilization in all organs.
A
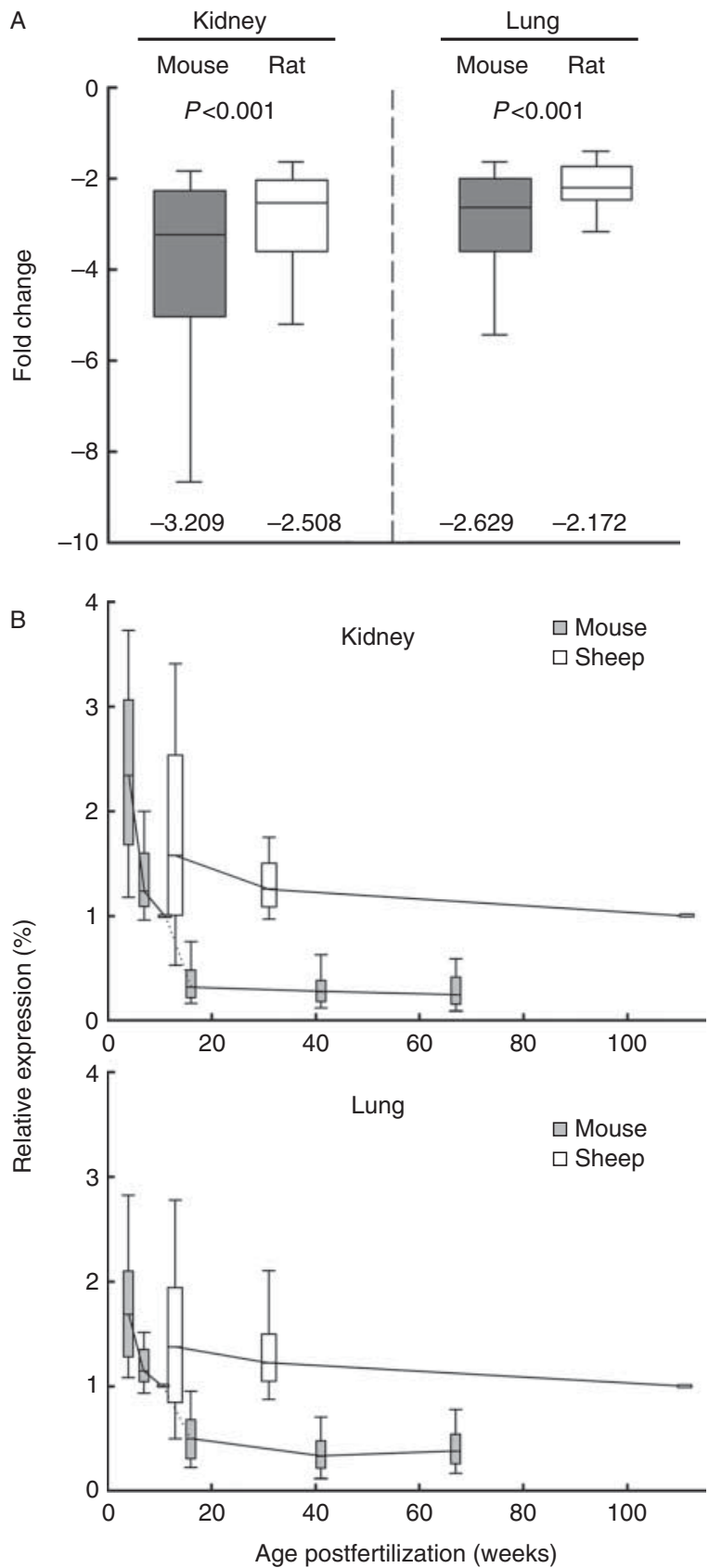

Figure 3

The pace of the multiorgan juvenile genetic program differs among species of different body size. (A) Mice vs rats. For the 235 genes that were downregulated with age in all organs studied in mouse and rat, we compared the fold change in expression from 1 to 4 weeks of age in the mouse vs 1 to 5 weeks in the rat by microarray. Negative values indicate decreasing expression. Boundaries of the box, 25th and 75th percentile; bars, 10th and 90th percentiles; central line segment, median. (B) Mice vs sheep. For genes that were commonly downregulated in all organs studied of all three species, we compared the expression patterns in mice and sheep by microarray. Age is expressed as weeks postfertilization to account for differences in the length of gestation. Relative expression was normalized to the young adult time point in each species (mouse, 11 weeks; sheep, 111 weeks). Dotted line separates data from different microarray experiments.

Published by Bioscientifica Ltd. 


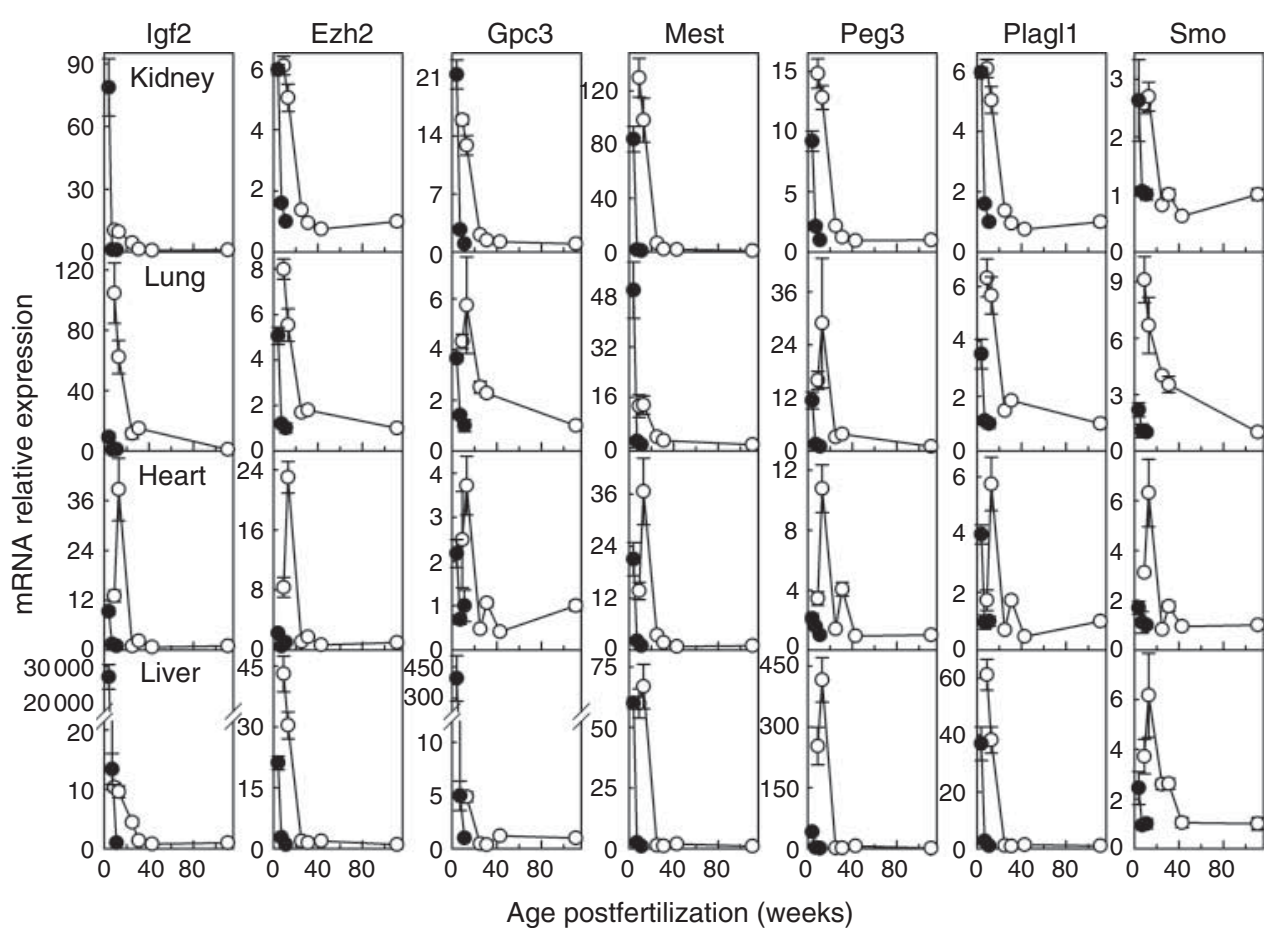

\section{Figure 4}

Downregulation of specific growth-regulating genes occurs more slowly in sheep (open circles) than in mice (closed circles). mRNA relative expression (mean \pm s.E.M.) was assessed by quantitative RT-PCR. Top to bottom row:

\section{Discussion}

In mammals, body growth is rapid in fetal and early postnatal life but then slows with age. We previously showed evidence that this growth deceleration in the mouse and rat is driven in part by a genetic program, which occurs in multiple tissues concurrently, and involves the downregulation of many growth-promoting genes (Finkielstain et al. 2009, Lui et al. 2010a). We first hypothesized that this juvenile growth-regulating genetic program is conserved in large mammals. Consistent with this hypothesis, we found that, in sheep, a large set of genes was coordinately regulated with age in both kidney and lung, an overlap far greater than would be expected by chance. The decreasing expression of multiple genes in both the sheep kidney and lung might occur because these genes are expressed primarily by a cell type common to both organs, such as stromal fibroblasts or endothelial cells. However, for at least some of the genes involved, Igf2, Mest, and Peg3, prior studies using in situ hybridization indicate that expression occurs primarily in organspecific parenchymal cells of the kidney and lung, as well as the heart and liver (Finkielstain et al. 2009). kidney, lung, heart, and liver. Relative expression was normalized to the young adult time point in each species (mouse, 11 weeks; sheep 111 weeks).

Bioinformatic analysis indicated that this overlap gene set was enriched for genes involved in cellular growth and proliferation. This enrichment occurred not only for genes downregulated with age, as had been observed previously in rodents, but also for genes upregulated with age, suggesting that growth deceleration may involve not only the downregulation of growth-promoting genes but also the upregulation of growth-inhibiting genes. We also found a striking similarity with the set of genes downregulated with age in juvenile rodents (Finkielstain et al. 2009, Lui et al. 2010a). These findings indicate that this juvenile multiorgan genetic program has been evolutionarily conserved over the $\sim 80$ million years since rodents and sheep diverged (dos Reis et al. 2012) and suggest that this program contributes to body growth regulation in both small and large mammals.

Mammalian body size varies enormously among species, from the Etruscan shrew, with an adult body mass $<2 \mathrm{~g}$ (Jurgens 2002) to the blue whale, with adult body mass $>100000 \mathrm{~kg}$ (Sears 2002). This wide variation in size is due in part to differences in the duration of body growth; somatic growth in small mammals generally lasts for weeks, whereas large mammals continue to grow for

Published by Bioscientifica Ltd. 
years. We hypothesized that the greater duration of growth in larger mammals occurs because the growthregulating genetic program plays out more slowly, with a more gradual decline in the expression of growthpromoting genes. We therefore compared the time course of this program in the mouse, rat, and sheep. For genes that were commonly downregulated in multiple organs of all three species, the decline occurred most rapidly in the mouse, the species with the smallest adult body size, and most slowly in the sheep, the largest species. Taken together, these findings are consistent with the hypothesis that the juvenile growth-regulating genetic program proceeds at a slower pace in larger mammals, allowing for more prolonged growth and greater adult body size.

The mechanisms that drive the downregulation of this set of growth-promoting genes in multiple organs are not well understood. In newborn rats, nutritional deprivation slows body growth and also slows progression of the program suggesting that this genetic program is not driven simply by time but is dependent on body growth (Marino et al. 2008, Lui et al. 2010a, Forcinito et al. 2011). A similar program in the skeletal growth plate appears also to be driven by the process of growth (Marino et al. 2008). However, it remains unknown whether the mechanisms underlying the common changes in gene expression are primarily local, intrinsic to the growing tissues, or systemic, for example, involving a circulating factor.

One of the limitations of the present study is that it involved only three mammalian species and a limited number of organs in each species. Because of practical considerations, microarray analysis was carried out in only two organs. Kidney and lung were chosen in order to make the most direct comparisons possible with the rodent microarray data (Finkielstain et al. 2009, Lui et al. 2010a). We then confirmed these findings using real-time RT-PCR in a limited number of genes by studying more time points in four organs chosen based on their varying embryonic origin, i.e., kidney and heart, which are derived from mesoderm, and liver and lung, which are of endodermal origin. The findings from these more detailed time courses and additional organs supported the hypothesis that the expression patterns in the sheep were similar to those reported in the mouse and rat, but with a more prolonged period of decline in the sheep. However, additional investigation involving a larger number of species, tissues, and genes would be important to confirm the current findings.

In conclusion we found evidence that the juvenile multiorgan growth-regulating genetic program recently described in the mouse and rat is conserved in the sheep.
We also showed evidence that this genetic program proceeds more slowly in larger mammals, consistent with our hypothesis that a slower pace of the program allows for more prolonged growth and therefore greater adult body size. Thus, evolutionary modulation of the pace of this genetic program may be responsible for the extensive variation in body size among mammalian species. A deeper understanding of these fundamental mechanisms regulating body growth may have important clinical implications; abnormalities of this program may contribute to unrestricted growth in malignant cells (Rezvani et al. 2012) and perhaps to disorders of childhood growth.

\section{Supplementary data}

This is linked to the online version of the paper at $\mathrm{http} / / / \mathrm{dx}$.doi.org/10.1530/ JME-13-0263.

\section{Declaration of interest}

The authors declare that there is no conflict of interest that could be perceived as prejudicing the impartiality of the research reported.

\section{Funding}

This work was supported by the Intramural Research Program of the Eunice Kennedy Shriver National Institute of Child Health and Human Development, National Institutes of Health. Sheep tissues used in the study were generated as part of NIH P01 HD44232 to V P. J B receives intramural NIH funding (HD000640) and $V \mathrm{P}$ receives extramural NIH funding (P01: HD044232).

\section{Acknowledgements}

The authors thank Dr Almudena Veiga-Lopez and Ms Carol Herkimer for assistance with procurement of tissues.

\section{References}

Chang M, Parker EA, Muller TJ, Haenen C, Mistry M, Finkielstain GP, Murphy-Ryan M, Barnes KM, Sundaram R \& Baron J 2008 Changes in cell-cycle kinetics responsible for limiting somatic growth in mice. Pediatric Research 64 240-245. (doi:10.1203/PDR.0b013e318180e47a)

Chen Y, Antoniou E, Liu Z, Hearne LB \& Roberts RM 2007 A microarray analysis for genes regulated by interferon- $\tau$ in ovine luminal epithelial cells. Reproduction 134 123-135. (doi:10.1530/REP-07-0387)

Diez-Tascon C, Keane OM, Wilson T, Zadissa A, Hyndman DL, Baird DB, McEwan JC \& Crawford AM 2005 Microarray analysis of selection lines from outbred populations to identify genes involved with nematode parasite resistance in sheep. Physiological Genomics 21 59-69. (doi:10.1152/physiolgenomics.00257.2004)

Finkielstain GP, Forcinito P, Lui JC, Barnes KM, Marino R, Makaroun S, Nguyen V, Lazarus JE, Nilsson O \& Baron J 2009 An extensive genetic program occurring during postnatal growth in multiple tissues. Endocrinology 150 1791-1800. (doi:10.1210/en.2008-0868)

Forcinito P, Andrade AC, Finkielstain GP, Baron J, Nilsson O \& Lui JC 2011 Growth-inhibiting conditions slow growth plate senescence. Journal of Endocrinology 208 59-67. (doi:10.1677/JOE-10-0302) http://jme.endocrinology-journals.org DOI: 10.1530/JME-13-0263
(C) 2014 Society for Endocrinology Printed in Great Britain
Published by Bioscientifica Ltd. 
Heinrichs C, Yanovski JA, Roth AH, Yu YM, Domene HM, Yano K, Cutler GB Jr \& Baron J 1994 Dexamethasone increases growth hormone receptor messenger ribonucleic acid levels in liver and growth plate. Endocrinology 135 1113-1118. (doi:10.1210/endo.135.3.8070354)

Hughes PC \& Tanner JM 1970 A longitudinal study of the growth of the black-hooded rat: methods of measurement and rates of growth for skull, limbs, pelvis, nose-rump and tail lengths. Journal of Anatomy $106349-370$

Jurgens KD 2002 Etruscan shrew muscle: the consequences of being small. Journal of Experimental Biology 205 2161-2166.

Kratochvilova M, Hyankova L, Knizetova H, Fiedler J \& Urban F 2002 Growth curve analysis in cattle from early maturity and mature body size viewpoints. Czech Journal of Animal Science 47 125-132.

Kwan AY \& Hartman ML 2007 IGF-I measurements in the diagnosis of adult growth hormone deficiency. Pituitary 10 151-157. (doi:10.1007/ s11102-007-0028-8)

Lui JC, Finkielstain GP, Barnes KM \& Baron J 2008 An imprinted gene network that controls mammalian somatic growth is down-regulated during postnatal growth deceleration in multiple organs. American Journal of Physiology. Regulatory, Integrative and Comparative Physiology 295 R189-R196. (doi:10.1152/ajpregu.00182.2008)

Lui JC, Chen W, Barnes KM \& Baron J 2010a Changes in gene expression associated with aging commonly originate during juvenile growth. Mechanisms of Ageing and Development 131 641-649. (doi:10.1016/ j.mad.2010.08.010)

Lui JC, Forcinito P, Chang M, Chen W, Barnes KM \& Baron J $2010 b$ Coordinated postnatal down-regulation of multiple growth-promoting genes: evidence for a genetic program limiting organ growth. FASEB Journal 24 3083-3092. (doi:10.1096/fj.09-152835)

MacKinnon KM, Burton JL, Zajac AM \& Notter DR 2009 Microarray analysis reveals difference in gene expression profiles of hair and wool sheep infected with Haemonchus contortus. Veterinary Immunology and Immunopathology 130 210-220. (doi:10.1016/j.vetimm.2009.02.013)

Manikkam M, Crespi EJ, Doop DD, Herkimer C, Lee JS, Yu S, Brown MB, Foster DL \& Padmanabhan V 2004 Fetal programming: prenatal testosterone excess leads to fetal growth retardation and postnatal catch-up growth in sheep. Endocrinology 145 790-798. (doi:10.1210/en. 2003-0478)

Marino R, Hegde A, Barnes KM, Schrier L, Emons JA, Nilsson O \& Baron J 2008 Catch-up growth after hypothyroidism is caused by delayed growth plate senescence. Endocrinology 149 1820-1828. (doi:10.1210/ en.2007-0993)

Pape L, Hoppe J, Becker T, Ehrich JH, Neipp M, Ahlenstiel T \& Offner G 2006 Superior long-term graft function and better growth of grafts in children receiving kidneys from paediatric compared with adult donors. Nephrology, Dialysis, Transplantation 21 2596-2600. (doi:10.1093/ndt/gfl119)

dos Reis M, Inoue J, Hasegawa M, Asher RJ, Donoghue PC \& Yang Z 2012 Phylogenomic datasets provide both precision and accuracy in estimating the timescale of placental mammal phylogeny. Proceedings. Biological Sciences 279 3491-3500. (doi:10.1098/rspb.2012.0683)

Rezvani G, Lui JC, Barnes KM \& Baron J 2012 A set of imprinted genes required for normal body growth also promotes growth of rhabdomyosarcoma cells. Pediatric Research 71 32-38. (doi:10.1038/pr.2011.6)

Schäfer R, Knauf U, Zweyer M, Högemeier O, de Guarrini F, Liu X, Eichhorn HJ, Koch FW, Mundegar RR, Erzen I et al. 2006 Age dependence of the human skeletal muscle stem cell in forming muscle tissue. Artificial Organs 30 130-140. (doi:10.1111/j.1525-1594.2006.00199.x)

Sears R \& Calambokidis J 2002 Update COSEWIC status report on the Blue Whale Balaenoptera musculus in Canada. In Committee on the Status of Endangered Wildlife in Canada, pp 1-32. COSEWIC: Ottawa, Canada.

Shrader AM, Ferreira SM, McElveen ME, Lee PC, Moss CJ \& van Aarde RJ 2006 Growth and age determination of African savanna elephants. Journal of Zoology 270 40-48. (doi:10.1111/j.1469-7998.2006.00108.x)

Stevens DG, Boyer MI \& Bowen CV 1999 Transplantation of epiphyseal plate allografts between animals of different ages. Journal of Pediatric Orthopaedics 19 398-403. (doi:10.1016/S0022-3476(85)80501-1)

Tanner JM \& Davies PS 1985 Clinical longitudinal standards for height and height velocity for North American children. Journal of Pediatrics 107 317-329. (doi:10.1016/S0022-3476(85)80501-1)

Winick M \& Noble A 1965 Quantitative changes in DNA, RNA, and protein during prenatal and postnatal growth in the rat. Developmental Biology 12 451-466. (doi:10.1016/0012-1606(65)90009-6)

Received in final form 5 February 2014

Accepted 28 February 2014 (c) 2014 Society for Endocrinology Printed in Great Britain
Published by Bioscientifica Ltd 